Acta vet. scand. 1973, 1't, 489—491.

Brief Communication

\title{
RESISTANCE OF PORCINE MYCOPLASMAS TO DRYING
}

The survival time of porcine mycoplasmas outside their natural habitat is of considerable interest, especially with regard to the question of preventive measures to be taken in order to avoid the introduction of such organisms in pig herds established under SPF-programmes. In a study on this problem Goodwin (1972) recorded a survival time of 1 month for Mycoplasma suipneumoniae stored in liquid medium at room temperature. Survival for 100 days was reported for storage in refrigerator.

The work here reported deals with experiments on the survival of porcine mycoplasmas after drying in air at room temperature.

Strains of all the known porcine mycoplasma species, cloned 3 times on solid medium, were included in the study. The strains were: a reference strain ${ }^{*}$ and 2 Danish isolates (M98 and M99) of Mycoplasma hyorhinis (M. hyor.); a reference $\operatorname{strain}^{\star \star}$ and 2 Danish isolates (Ms5 and Ms6) of Mycoplasma suipneumoniae (M. suip.); a reference strain ${ }^{\star \star \star ~(S 39) ~ o f ~ A c h o l e p l a s m a ~ g r a n u-~}$ larum (A. gran.); a Danish isolate (M60) of Mycoplasma hyosynoviae (M. hyosyn.); a strain of Mycoplasma flocculare (M. floc.) designated Ms42 (Meyling \& Friis 1972). By the growth and metabolic inhibition tests (Friis 1971a) M98, M99, Ms5, and Ms6 were indistinguishable from the respective reference strains, and M60 from the reference strain of M.hyosyn. (S16, Ross \& Karmon 1970). Media previously described by Friis $(1970,1971 \mathrm{~b})$ were used for cultivation. Well-growing broth cultures in the final stage of the logarithmic growth phase were diluted about 10 times in pure broth, and from each strain 1 drop was deposited

* and * Type NCTC 10,121 and Type NCTC 10,110, respectively, Mycoplasma Reference Laboratory, Colindale, England.

** Obtained from Dr. D. H. Roberts, Central Veterinary Laboratory, Weybridge, England. The strain originates from Dr. W. P. Switzer, Iowa State University, Ames, Iowa, USA. 

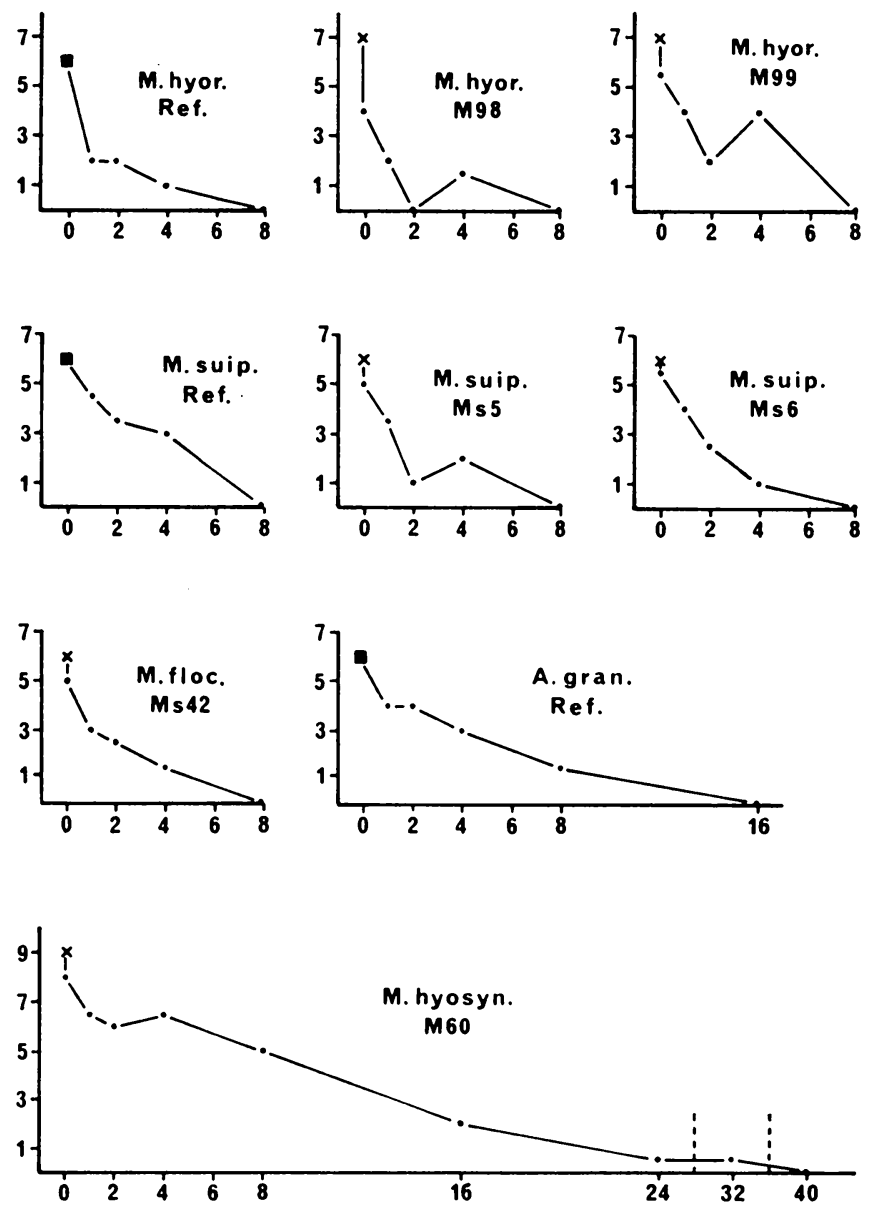

Figure 1. Resistance to air-drying of porcine mycoplasmas suspended in broth.

Ordinates: Endpoints of growth (log. of reciprocal) in 10-fold dilutions of the resuspended dried cultures. Abcisses: Days after drying when cultivation was made.

Symbol " $\times$ “ indicates control titer before drying. Symbol " $\square$ “ indicates coincidence of control titer with titer immediately after drying. Ref. = reference strain (see text).

on each of a number of sterile coverslips (Chance, Microscope Cover Glasses, $9 \times 33 \mathrm{~mm}$ ) and spread over an area of about $1 \mathrm{~cm}^{2}$. The coverslips were left on the bench (sun excluded) at room temperature $\left(20^{\circ}-25^{\circ} \mathrm{C}\right)$, open to evaporation. When thoroughly dry (1.0-1.5 hrs.) each coverslip was placed in a 
separate, empty plastic tube, which was sealed with a rubber stopper. The tubes were stored in the dark at room temperature. Cultures were made from coverslips before drying and at suitable intervals afterwards, i.e., immediately after drying, after 1, 2, 4, 8, and 16 days, and if found necessary (M.hyosyn.) even later. The cultivation was carried out in the following way: to each tube containing a coverslip with dried culture $1.8 \mathrm{ml}$ of medium was added, and $5 \mathrm{~min}$. later 10 -fold dilutions were prepared. The cultures were incubated in a revolving drum at $37^{\circ} \mathrm{C}$ and the titers read at the end of the growth period. Colourshift of the phenol red in the broth was taken as indication of growth. With the exception of the controls all the experiments were made in duplicate.

The results are given in Fig. 1. As will be seen, M. hyor., M. suip., M.floc., and A. gran. did not survive air-drying beyond approx. 1 week. In this connection a survival time of about 1 month as noted for the strain of M. hyosyn. is remarkable.

\section{N. F. Friis}

The State Veterinary Serum Laboratory, Copenhagen, Denmark.

\section{REFERENCES}

Friis, N. F.: A new porcine mycoplasma species: Mycoplasma suidaniae. Acta vet. scand. 1970, 11, 487-490.

Friis, N. F.: Mycoplasmas cultivated from the respiratory tract of Danish pigs. Acta vet. scand. 1971a, 12, 69-79.

Friis, N. F.: Sensitivity of Mycoplasma suipneumoniae to penicillin-G. Acta vet. scand. $1971 \mathrm{~b}, 12,120-121$.

Goodwin, R. F. W.: The survival of Mycoplasma suipneumoniae in liquid medium, on solid medium and in pneumonic tissue. Res. Vet. Sci. 1972, 13, 203-204.

Meyling, A.\& N. F. Friis: Serological identification of a new porcine mycoplasma species, M. flocculare. Acta vet. scand. 1972, 13, 287-289.

Ross, R. F. \& Judith A. Karmon: Heterogeneity among strains of Mycoplasma granularum and identification of Mycoplasma hyosynoviae, sp.n. J. Bact. 1970, 103, 707-713.

(Received May 28, 1973).

Reprints may be requested from: N. F. Friis, State Veterinary Serum Laboratory, Bülowsvej 27, DK-1870 Copenhagen V, Denmark. 